\title{
A Summary of Reactive Power Optimization Algorithms
}

\section{Dongyue Zhang ${ }^{1, a}$, Kewen Wang ${ }^{1, b}$}

\author{
${ }^{1}$ School of Electrical Engineering, Zhengzhou University, Zhengzhou 450001, China. \\ achun_he_jing_ming@163.com, ${ }^{\mathrm{b}} 1196213034 @ q q . c o m$
}

Keywords: power system, reactive power optimization, traditional algorithms, artificial intelligence optimization algorithm, hybrid optimization algorithm.

\begin{abstract}
In order to comprehensively generalize the research status quo, achievements as well as deficiencies on the power system reactive power optimization algorithms, a universal reactive power optimization model was introduced in this paper, and a variety of common traditional optimization algorithms, artificial intelligence optimization algorithms used in current power system reactive power optimization were summarized, including linear programming, nonlinear programming, dynamic programming, modern heuristic search, expert system method, etc. The principle, advantages and disadvantages of each algorithm were analyzed in this paper, at the same time, several improved optimization algorithms which aimed at the flaws of each basic optimization algorithm were also introduced. Finally, the paper listed several effective hybrid optimization algorithms, and offered a proposal on the research direction of reactive power optimization in the future.
\end{abstract}

\section{Introduction}

The math model of power system optimal power flow (OPF) can be described as: when the structure parameters and load of the system had been set, adjust the control variables to find the power flow distribution that can satisfy all specified constraints, and make a certain performance indicators or the objective function of the system to achieve the best ${ }^{[1]}$. Reactive power optimization problem is a branch of OPF, it contains multiple constraints and control variables, meanwhile, the control variable includes continuous variables and discrete variables. The terminal voltage of the generator belongs to continuous variables, while capacitor switching and transformer tap changing are discrete variables. So the reactive power optimization problem is a mixed nonlinear programming problem.

With the scale of power grid is expanding,the complexity of it's structure and the degree of power market are increasing, how can the power system run safely, stably and economically is more and more noticeable. Meanwhile, the reactive power optimization problems involved in all areas of the system operation, so it is a problem that scholars at home and abroad both keen to research. Usually, the reactive power optimization research includes the establishment of the optimization model and the determination of the optimization algorithm.

Reactive power optimization model. Determining the objective function and constraint conditions is the key to establish reactive power optimization model. The objective function decides the optimization strategy. Common objective functions are: voltage quality optimal, active power loss minimum, the sum of line loss and equipment adjusting cost minimum, etc. Among them, the model whose objective function is making the active loss minimum is most widely used.

$$
\left.\begin{array}{ll} 
& \min f(u, x) \\
\text { s.t. } & g(u, x)=0 \\
& h(u, x) \leq 0
\end{array}\right\} .
$$

In formula (1):

$u$ - control variable, it usually includes the active power of all generator sets except the balance node, the reactive power of all generators and reactive power compensation devices, adjustable transformer tap positions, etc.

$f(u, x) \longrightarrow$ objective function of reactive power optimization;

$g(u, x)$ _equality constraint,the fundamental power flow equation; 
$h(u, x)$ — inequality constraint that control variables and state variables should satisfy.

Reactive power optimization algorithm. With the rapid development of modern power system and computer technology, new reactive power optimization algorithms are emerging in an endless stream. In general, reactive power optimization algorithms that currently exist can be roughly divided into the following three categories.

Traditional optimization algorithm. Traditional optimization algorithm is produced based on the principle of operational research. The math model of this kind of algorithm is accurate and its constraint conditions are clear. The current solution can be improved continuously along a certain path from an initial point, and converge to the optimal solution eventually. Common traditional reactive power optimization algorithms are linear and nonlinear programming method, dynamic programming method as well as mixed integer programming method which handle the linear and nonlinear problems separately, etc.

The basic principle of linear programming method is:use Taylor formula to expand the objective functions and constraint conditions respectively, keep only the first degree terms, thus the nonlinear problem can be linearized near the initial point and reactive power optimization problem can be solved with the method of linear approximation. This method is very mature now. It had been proved that using this method to solve the problem of reactive power optimization can achieve a good effect in literature [2]. Methods such as sensitivity analysis method, interior-point method are all common linear programming. Among them, sensitivity analysis method is the most representative ${ }^{[3]}$.

The principle of sensitivity analysis method is: first, using Jacobi matrix in the flow calculation of Newton Raphson algorithm to get the sensitivity matrix of state variables to control variables, then solve the problem with dual linear programming method based on this. It's easy to introduce various constraint conditions with the sensitivity matrix and can get ideal result of the reactive power optimization. However, this method involves the inversion of high-order Jacobi matrix, large amount of calculation, memory and time are demanded. Besides, the application of simplified assumption has also led to a decline of the calculation precision and the convergence speed of the algorithm.

The method of Jacobi matrix transformation is used in literature[4],only with one matrix transformation can we get the matrix of loss sensitivity coefficient and relative sensitivity coefficient. Meanwhile, the computing speed is greatly accelerated especially for large-scale systems.In literature [5], the state variables are monitored directly by the sensitivity matrix of the objective function to control variables.So the oscillation was avoided because the iteration is always towards the direction which can make the value of the objective function decrease.Using "perturbation" method to get sensitivity matrix was firstly put forward in literature[6],the convergence of this algorithm is relatively good and need less amount of calculation than methods using inverse matrix of augmented Jacobi.

The basic idea of interior-point method ${ }^{[7-11]}$ is: start from a point in the feasible region and along the path which make the value of the objective function decrease fastest until achieve the optimal solution.Meanwhile, the whole optimization process of this algorithm must be within the feasible region.

Original-dual affine-scaling interior point method is the most potential interior-point method, it also known as path following method. Path following method has good robustness and convergence, and the computing time won't increase noticeably with the scale of the system ${ }^{[12]}$. The experiment result of literature[13] shows that the iterative times of path following method is stable and affected by the scale of system not obviously because this method is a multinomial time complexity algorithm. But it's initial point have to be in the feasible region and the optimization process has to along the original-dual path,even the step size of the control variable will effect the process. These defects are remedied by method in literature[14], with this method, the initial point is not confined to the feasible region and the optimization process is not necessary to along the original-dual path but still can converge to the optimal solution, and the convergence performance is stable.

Linear programming method has a simple mathematical model and a high speed. Besides, the scale of the system it can calculate is nearly unrestricted because it has developed very perfect. However, 
this method is a approximative linearization for the mixed nonlinear programming model and a continuous for the discrete variable, so the final result has a certain error.

Due to reactive power optimization is a nonlinear problem, the earliest method used to solve the problem is nonlinear programming method. The most representative nonlinear programming methods are: reduced gradient method, Newton method and quadratic programming method.

Reduced gradient method is based on Newton power flow calculation that of polar form. It deal with quality and inequality constraints respectively by using Lagrange multiplier method and penalty function, thus it has a first order convergence. This method is the first relatively successful optimal power flow algorithm ${ }^{[15]}$.

The algorithm of reduced gradient method is simple, the program design theme is clear. It has no strict requirement for initial value and need not very big storage. It is easy to realize and convenient to solve large-scale OPF problems. But the algorithm is prone to appear sawtooth and have a bad convergence performance. Every movement need to compute the current trend, so the demand for calculation and time is large. In addition, the convergence speed is closely related to the size of the penalty factor when using penalty function to deal with inequality constraints. Method in literature[16] use Fletcher-Powell algorithm to fix the step size which improves the convergence properties.

Newton method uses not only the Jacobi matrix produced by the first derivative of the system's power flow equation but also the Hansen matrix produced by the second derivative of the objective function, in another words, this method concerns the trend of gradient changing. So it has a more reasonable direction for search and a convergence rate of second-order. Besides, the high sparsity of Jacobi and Hansen matrix decrease the computing amount greatly. But solving the Hansen matrix is time consuming. At present, the key problem that restrict the applying of Newton method is how to select the working inequality constraints accurately. Method in literature [17] can deal with the inequality constraints well, at the same time, it successfully avoid the problem of selecting the working inequality constraints in Newton method.

Quadratic programming method is a kind of special nonlinear programming method, because it can only solve problems whose objective functions are quadratic and constraint conditions are linear. It is widely used because of its ideal astringency. The author of literature[18] introduced people a breakthrough method which optimize by transforming the original nonlinear model into several sub-problems of quadratic programming with decomposition method.

Methods above each have its own advantages and disadvantages, but all of them have a similarity that considering discrete variables as continuous variables at the beginning, then give back them to the nearest discrete points after the optimization. That is to say, we can only approach rather than achieve the optimal solution. Even some giving back may cause some constraint conditions off-limits and become non-feasible solutions.In order to solve this problem, mixed integer programming method deal with integer and continuous variables separately. But the separate lead to a bad integrality, a complex calculation process, a vast number of calculations and a slow convergence. Meanwhile, the calculation process prone to oscillations since the nonlinear function relation of reactive power and voltage.

Conclusion of literature [19] shows that solving discrete variable problems with quadratic penalty function makes the solution much closer to the optimum one and the convergence rate is more ideal. However, this method needs to introduce penalty function and select penalty factors of right size at the appropriate time. Besides, there are many parameters need to be set manually. Method in literature[20] guarantees a feasible optimum solution by adjusting the returning direction of discrete variables with experts' knowledge.

The principle of dynamic programming is that divide the problem into several connected stages in chronological or space order firstly and make decisions for every stages, then balance all the stages to get a optimal solution. Dynamic programming have its unique advantages compared with linear and nonlinear programming: Globally optimal solution usually can be get without the objective function and constraint conditions observing the linear and convexity strictly, meanwhile, its convergence is good. However, too many variables lead to a more complex modeling and lower speed as well as "dimension disaster",thus the application of this method in practical engineering was restricted. 
From the analysis of various algorithms above we know that all conventional optimization algorithms have some common flaws: difficult to apply to practical because of the high requirement for the accuracy of the math model; can't solve the issue of discrete variables very well; have high requirement for the initial point; "dimension disaster". In order to solve these problems, of course it benefit from the rapid development of artificial intelligence and computer technology, various artificial intelligence algorithm emerge as the times require.

Artificial intelligence algorithm. The most common artificial intelligence algorithm are modern heuristic search algorithm, expert system, artificial neural network ,etc.

Tabu Search method is the most successful modern heuristic search algorithm. It use Tabu table to record "movements" that have been iterated to guide the direction of next step in case repeated search.However, in order to grasp every potential "movement" that may generate optimal solution, rules of "amnesty"is set in Tabu search method. Once the rule was satisfied, the movement is permitted even it is in the Tabu table. This method need less iterations but have an ideal convergence rate. However, the rate of convergence and the quality of the final solution are closely related to the initial value because it use single point search. Besides, the global search ability of Tabu search is poor, and the searching efficiency decreases with the increasing of Tabu list's scale.

Improved Tabu algorithm in literature [21] have no specific requirement for the initial value but get a higher speed. Method in literature [22]improves the global search ability by taking measures such as: adaptive step length, no Tabu table, add stopping criteria .etc.

In 1975, the American scholar J.Holland put forward a random search method that simulating the biology evolution, that is genetic algorithm (GA). GA has a great ability of global search because it has several initial points and can search in multiple directions. This method can avoid "dimension disaster" and has strong robustness as well as parallel computing ability. However, its local searching ability, convergence and resolution are poor.

Literature[23] introduced niche genetic algorithm aimed at the flaws of common GA, it can overcome the problems of poor local optimization ability(prone to "premature") and convergence at later period, meanwhile, obtain the optimal solution quickly and efficiently. In addition to GA, there are many similar simulated evolutionary algorithms, such as artificial fish algorithm, ant colony algorithm, artificial colony algorithm, evolutionary programming, collaborative evolution method, multi-agent algorithm, etc.

Simulated annealing algorithm can solve combined optimization problems with constraint conditions well, even it is able to converge to the global optimal point with a probability close to 1 . Meanwhile, it is easy to realize. However, there may be repeated searching because it has no memory function. And algorithm becomes more complex, calculation speed becomes lower with the scale of the system expanding. In addition, the improper selection of the initial temperature, cooling speed, termination criterion of the annealing scheme can also lead to bad convergence and low speed, but these conditions are difficult to satisfy in practice. The improved simulated annealing algorithm introduced by literature[24] not only avoid the repeated optimization to some extent but also make up the weakness of short of local optimization ability.

Expert system is a kind of computer program that based on the conventional algorithm. It is able to simulate human experts to analyze practical problems and make reasonable decisions with expert knowledge, experience, historical data that widely collected. Its characteristic was greatly optimized because the widely reference of expert knowledge and experience. But there are still many problems meed to be solved, such as the interfaces are not friendly enough, the means to acquire knowledge are not flexible, etc.

Artificial neural network model is build according to the human cranial nerve network,and the independent computing among all the neurons makes parallel computing is feasible, so this method is also called parallel distributed processing model. Due to its strong collectively operating ability, autonomous learning ability and good convergence, predictability, guidance, and flexibility, artificial neural network is used in power system operation. However, it can't be applied to large-scale systems' online real-time controlling because appropriate learning strategies haven't been fond and easy into local optimal solution. 
In addition to the algorithms that have been listed, there are many other common artificial intelligence algorithms like immune algorithm, particle swarm optimization algorithm, Box algorithm, difference evolution algorithm, differential evolution method, hybrid leapfrog algorithm, simulating plant growth algorithm, decomposition-coordination algorithm and so on.

Hybrid optimization algorithm. Because conventional methods usually have their own disadvantages, scholars put forward hybrid optimization algorithm to get more perfect optimization result. This method is to combine two or more kinds of algorithms together, and use advantages of one method to offset the disadvantages of other methods.

For example, literature[25] uses the combination of an improved interior-point method and genetic algorithm to realize the reactive power optimization. It makes up the corresponding defects of GA with nonlinear interior-point method's advantages of strong robustness, fast calculation and good convergence. In order to solve the problem that the action number of discrete equipment is limited, literature[26] put forward the idea of integrating immune genetic algorithm and non-linear interior point method. Experiment proves that the speed and accuracy of the hybrid algorithm are improved.

\section{Summary}

First, this paper introduced the general model of reactive power optimization. Second, the principles as well as the advantages and disadvantages of various basic optimization algorithm were analyzed and summarized.In addition, some improved algorithms aimed at the defects of basic optimization algorithms had been listed and their properties are obviously better than the original algorithms. Last, hybrid optimization algorithm that can satisfy the high expectation of speed, convergence and precision was introduced.

Nowadays, renewable energy generation and UHV transmission is more and more popular, but the access of them will change the characteristics of the power system greatly,then the reactive power optimization algorithms can keep pace with the times and constantly adapt to the developing power system.

\section{Acknowledgments}

Corresponding author: Dongyue Zhang, Email:chun_he_jing_ming@163.com

\section{References}

[1] Heng Chen, Steady-State Analysis of Power System, China Electric Power Press, Beijing, 1995.

[2] Hobson E, et al, Linear programming for power system real time control calculation, IFAS Conference Melbourne, 1977.

[3]Boming Sun, Shousun Chen, Higher Power Network Analysis, Tsinghua University Press, Beijing, 1996.

[4] Yiqing Zhang, Application of analysis and power grid planning in the EMS and DMS,Association of Computer Users of Hunan. 3 (1994) 156-166.

[5] Youxin Zhao, Quoyu Xu, Analyze and calculate the optimal control problem of reactive power and voltage with the sensitivity method, Journal of Chongqing University. 2 (1985) 1-11.

[6] Jakun He, Qingan Zhang, A power system var comprehensive optimal dispatching using overall sensitivity analysis method, Proceeding of the CSEE. 5 (1985) 36-46.

[7] Juan Yu, Wei Yan, Guoyu Xu, et al, A new model of reactive optimization based on predictor primal dual interior point method, Proceeding of the CSEE. 25 (2005) 146-151.

[8] Fang Liu,Wei Yan, David C.Yu, Mixed strategy of reactive power optimization based on genetic algorithm and interior-point method, Proceeding of the CSEE. 25 (2005) 67-72. 
[9] Yongfu Wang, Boming Zhang, Hongbin Sun, et al, Real-time balance trading algorithm based on interior-point method, Power System Technology. 28 (2004) 48-50.

[10] Xiufan Le, Zhencheng Qin, Bo Yang, Opitimal power flow based on improved multiple centrality-correction interior point method, Power System Technology. 29 (2005) 47-52.

[11] Guanglin Cai, Hua Wei, An interior point optimal power flow algorithm based on nonlinear complementary method, Power System Technology. 29 (2005) 21-26.

[12] Jabr R A, Coonick A H, Cory B J, A primal-dual interior point method for power flow dispatching, IEEE Transactions on Power System. 17 (2002) 654-662.

[13] MingBo Liu, Xuejun Chen, Prime-dual affine scaling interior point method based reactive power optimization in power systems, Power System Technology. 22 (1998) 21-28.

[14] MingBo Liu, Xuejun Chen, Improved interior point method for reactive power optimization in power systems, Automation of Electric Power Systems. 22 (1998) 33-36.

[15] H. W. Dommel, W. F. Tinney, Optimal power flow solutions, IEEE Transactions on Power Apparatus and Systems. 3 (1962) 431 447.

[16] Albert M. Sasson, Combined use of the parallel and Fletcher-Powell non-linear for optimal load flows, IEEE Transactions on Power Apparatus and Systems. 10 (1969) 1530-1537.

[17] Yongping Zhang, Xiaojiao Tong, Fuli Wu, et al, Study on semi-smooth Newton optimal power flow algorithm based on nonlinear complimentary problem function, Proceeding of the CSEE. 9 (2004) 130-135.

[18] R. C. Burchett, H. H. Happ, D. R. Vierath, Quadratically convergent optimal power flow, IEEE Transactions on Power Apparatus and Systems. (1984) 3267-3276.

[19] Ying Cheng, Mingbo Liu, Design and application of electric power network stability real-time monitoring software, Automation of Electric Power Systems. 5 (2001) 23-25.

[20] Mingtian Fan, Research on discrete reactive power optimization algorithm of power system, Tsinghua University.1995.

[21] Ning Xiong, Ken Chen, The application of the improved tabu algorithm in reactive power optimization, Electricity of Jiangxi. 30(2006)24-30.

[22] Hongzhang Wang, Xinyin Xiong, Yaowu Wu, Power system reactive power optimization based on modified Tabu search algorithm, Power System Technology. 26 (2002) 15-18.

[23] Hongwei Zhang, Lijun Wu, Yi Ma, Analysis of reactive power optimization in power system based on niche genetic algorithm, Water Resources and Power. 27 (2009) 187-199.

[24] Dexiang Jia, Guoqing Tang, Jing Han, Reactive power optimization of power system based on modified simulated annealing algorithm, Relay. 32 (2004) 32-35.

[25] Xiaoyan Qiu, Zijian Zhang, Xingyuan Li, Multi-objective reactive power optimization based on improved genetic-interior point algorithm, Power System Technology. 33 (2009) 27-31.

[26] Bin Cheng, Fang Liu, Wei Yan, et al, Hybird intelligent method for dynamic reactive power optimization, Journal of Chongqing University. 30 (2007) 22-27. 